\title{
Gravitational waves from first-order phase transition and domain wall
}

\author{
Ruiyu Zhou, Jing Yang and Ligong Bian ${ }^{1}$ \\ Department of Physics, Chongqing University, \\ Chongqing 401331, China \\ E-mail: zhoury@cqu.edu.cn, 201927021002@cqu.edu.cn, 1gbycl@cqu.edu.cn
}

ABSTRACT: In many particle physics models, domain walls can form during the phase transition process after the breakdown of the discrete symmetry. Utilizing the $\mathbb{Z}_{3}$ symmetric complex singlet scalar extension of the Standard Model, we study the gravitational waves produced by the strongly first-order electroweak phase transition and the domain wall decay. The gravitational wave spectrum is of a typical two-peak shape. The high frequency peak corresponding to the strongly first-order electroweak phase transition is able to be probed by the future space-based interferometers, and the low frequency peak coming from the domain wall decay is far beyond the capability of the current Pulsar Timing Arrays, and future Square Kilometer Array.

Keywords: Beyond Standard Model, Thermal Field Theory

ArXiv EPrint: 2001.04741

\footnotetext{
${ }^{1}$ Corresponding author.
} 


\section{Contents}

1 Introduction 1

2 The $\mathbb{Z}_{3}$ symmetric complex singlet extended Standard Model 2

3 Electroweak phase transition $\quad 4$

3.1 Phase transition dynamic 4

$\begin{array}{lll}3.2 & \mathrm{BNPC} \text { and the strongly first-order EWPT condition } & 6\end{array}$

4 Gravitational wave $\quad 6$

$\begin{array}{lll}4.1 & \text { Gravitational waves from the first-order EWPT } & 7\end{array}$

4.2 Gravitational waves from domain wall decay 9

4.3 Gravitational waves from strongly first-order EWPT and domain wall 11

5 Conclusion and discussion $\quad 12$

$\begin{array}{lr}\text { A Vacuum structures and phase transition types } & 12\end{array}$

$\begin{array}{ll}\text { B Electroweak sphaleron } & 13\end{array}$

\section{Introduction}

The observation of black hole binary merger [1] and the approval of Laser Interferometer Space Antenna (LISA) by European Space Agency [2] raise growing interest in the gravitational wave study. The strongly first-order electroweak phase transition (EWPT), as one of the crucial ingredients for the electroweak baryogenesis [3], can produce a stochastic gravitational wave background with the peak frequency locating within the sensitivity region of the LISA [4]. The phase transition in the Standard model with the observed Higgs mass is confirmed to be cross-over [5], and a first-order EWPT usually require extension of the Higgs sector [6]. The Higgs pair searches at future collider can serve as a probe of the phase transition parameters of new physics models [7]. Therefore, it is possible to probe the strongly first-order EWPT with complementary searches of colliders and gravitational waves [8-13]. The cosmic phase transition with spontaneously broken of a discrete symmetry may create domain walls [14], which can be unstable to avoid overclose the Universe [15-17] by including approximately and explicitly broken terms in the models. Different from literatures, we are going to study domain walls formation and decay after a strongly first-order EWPT, and the produced gravitational waves during the process. The simplest way to obtain a first-order EWPT is to extend the Standard model with a singlet scalar, which belongs to the tree-level renormalizable model class where the first-order EWPT is triggered by a tree-level barrier [18]. In this work, we study the complex singlet 
scalar extended Standard Model with a $\mathbb{Z}_{3}$ symmetry, which is well motivated for the dark matter and neutrino physics [19-23]. The gravitational wave produced at the strongly firstorder phase transition within the model has been studied in refs. [24-26]. ${ }^{1}$ Different from previous studies, we study the gravitational waves produced from the strongly first-order electroweak phase transition, and domain wall decay. We first check the strongly firstorder EWPT condition by evaluating the baryon number preservation criterion (BNPC), and then study the relation among the criterion and gravitational wave parameters, i.e, the latent heat, and the inverse duration of the phase transition. After that, we study the possibility to probe the gravitational wave from the domain wall decay at the European Pulsar Timing Array (EPTA [28]), the Parkes Pulsar Timing Array (PPTA [29]), the International Pulsar Timing Array (IPTA [30]), and the Square Kilometer Array (SKA) [31]. Finally, we present a novel gravitational wave spectrum, with one peak locating within sensitivity range of these Pulsar Timing Arrays and another peak can be probed by future space-based interferometers.

\section{The $\mathbb{Z}_{3}$ symmetric complex singlet extended Standard Model}

In this work, we consider the model with the scalar potential being given by,

$$
V=\mu_{H}^{2}|H|^{2}+\lambda_{H}|H|^{4}+\mu_{S}^{2}|S|^{2}+\lambda_{S}|S|^{4}+\lambda_{\mathrm{SH}}|S|^{2}|H|^{2}+\frac{\mu_{3}}{2}\left(S^{3}+S^{\dagger 3}\right) .
$$

The cubic $\mu_{3}$ term breaks the global U(1) $S \rightarrow e^{i \alpha} S$ symmetry with a remanent unbroken $\mathbb{Z}_{3}$ symmetry. We expand the scalar fields around their classical backgrounds as follows,

$$
H=\left(\begin{array}{c}
G^{+} \\
\frac{h+i G^{0}}{\sqrt{2}}
\end{array}\right), \quad S=\frac{s+i \chi}{\sqrt{2}},
$$

and obtain the tree-level potential,

$$
\begin{aligned}
V_{0}(h, s, \chi)= & \frac{\lambda_{H}}{4} h^{4}+\frac{\lambda_{S}}{4} s^{4}+\frac{\lambda_{S}}{4} \chi^{4}+\frac{\lambda_{\mathrm{SH}}}{4} h^{2} s^{2}+\frac{\lambda_{\mathrm{SH}}}{4} h^{2} \chi^{2}+\frac{\lambda_{S}}{2} s^{2} \chi^{2} \\
& +\frac{\mu_{3}}{2 \sqrt{2}} s^{3}-\frac{3 \mu_{3}}{2 \sqrt{2}} s \chi^{2}+\frac{\mu_{H}^{2}}{2} h^{2}+\frac{\mu_{s}^{2}}{2} s^{2}+\frac{\mu_{s}^{2}}{2} \chi^{2} .
\end{aligned}
$$

Considering the stationary point conditions,

$$
\left.\frac{d V_{0}(h, s, \chi)}{d h}\right|_{h=v}=0,\left.\frac{d V_{0}(h, s, \chi)}{d s}\right|_{s=v_{s}}=0,
$$

we get $\mu_{H}^{2}=-\lambda_{H} v^{2}-\frac{1}{2} \lambda_{\mathrm{SH}} v_{s}^{2}, \mu_{s}^{2}=-\lambda_{S} v_{s}^{2}-\frac{1}{2} \lambda_{\mathrm{SH}} v^{2}-\frac{3 \sqrt{2}}{4} \mu_{3} v_{s}$. The Higgs mass matrix is then given by,

$$
M^{2}=\left(\begin{array}{cc}
2 \lambda_{H} v^{2} & \lambda_{\mathrm{SH}} v v_{s} \\
\lambda_{\mathrm{SH}} v v_{s} & 2 \lambda_{S} v_{s}^{2}+\frac{3}{2 \sqrt{2}} \mu_{3} v_{s}
\end{array}\right) .
$$

\footnotetext{
${ }^{1}$ For a gravitational wave study in complex singlet model connecting with axion-like particle, we refer to ref. [27].
} 
The stationary point can be a minimum when one have a positive determination of the zero temperature Hessian matrix, which yields:

$$
\lambda_{H}>0, \quad 8 v_{s} \lambda_{S}+3 \sqrt{2} \mu_{3}>0, \quad 8 v_{s} \lambda_{H} \lambda_{S}-2 v_{s} \lambda_{\mathrm{SH}}^{2}+3 \sqrt{2} \lambda_{H} \mu_{3}>0 .
$$

Introducing the rotation matrix $R=((\cos \theta, \sin \theta),(-\sin \theta, \cos \theta))$, and rotating into the mass basis through

$$
\left(\begin{array}{l}
h_{1} \\
h_{2}
\end{array}\right)=\left(\begin{array}{cc}
\cos \theta & -\sin \theta \\
\sin \theta & \cos \theta
\end{array}\right)\left(\begin{array}{l}
h \\
s
\end{array}\right),
$$

one has,

$$
\begin{aligned}
& m_{h_{1}}^{2}=\frac{1}{4} v_{s}\left(8 v_{s} \lambda_{S}+3 \sqrt{2} \mu_{3}\right) \sin \theta^{2}+2 v \cos \theta\left(v \lambda_{H} \cos \theta+v_{s} \lambda_{\mathrm{SH}} \sin \theta\right) \\
& m_{h_{2}}^{2}=\frac{1}{4} v_{s}\left(8 v_{s} \lambda_{S}+3 \sqrt{2} \mu_{3}\right) \cos \theta^{2}-2 v v_{s} \lambda_{\mathrm{SH}} \cos \theta \sin \theta+2 v^{2} \lambda_{H} \sin \theta^{2}
\end{aligned}
$$

The mixing angle $\theta$ can be expressed as follows,

$$
\tan 2 \theta=\frac{\lambda_{\mathrm{SH}} v v_{s}}{\lambda_{H} v^{2}-\lambda_{S} v_{s}^{2}-\frac{3}{4 \sqrt{2}} \mu_{3} v_{s}} .
$$

For our study, we consider $h_{1}=h_{S M}$, and $m_{h_{2}}>m_{h_{1}}$. After breakdown of the electroweak symmetry together with the $\mathbb{Z}_{3}$ symmetry, the $S \rightarrow S^{\dagger}$ is equivalent to $\chi \rightarrow-\chi$. Different from refs. $[25,26]$, we do not consider $\chi$ as dark matter in this work. The mass of the pseudo-Goldstone $\chi$ is given by,

$$
m_{\chi}^{2}=-\frac{9}{2 \sqrt{2}} \mu_{3} v_{s}
$$

which is proportional to $\mu_{3}$. Requiring the electroweak symmetry together with the $\mathbb{Z}_{3}$ symmetry broken vacuum being the global minimum, one has

$$
m_{\chi}^{2}<\frac{9 m_{h_{1}}^{2} m_{h_{2}}^{2}}{m_{h_{1}}^{2} \cos ^{2} \theta+m_{h_{2}}^{2} \sin ^{2} \theta},
$$

which severely constrain the relation among $m_{h_{2}}, m_{\chi}$, and $\theta$. The relation among the interaction couplings, Higgs masses, VEVs, and mixing angle $\theta$ are:

$$
\begin{aligned}
\lambda_{H} & =\frac{m_{1}^{2}+m_{2}^{2}+\left(m_{1}^{2}-m_{2}^{2}\right) \cos 2 \theta}{4 v^{2}} \\
\lambda_{S} & =\frac{3\left(m_{1}^{2}+m_{2}^{2}\right)+2 m_{\chi}^{2}+3\left(m_{2}^{2}-m_{1}^{2}\right) \cos 2 \theta}{12 v_{s}^{2}} \\
\lambda_{\mathrm{SH}} & =\frac{\left(m_{1}^{2}-m_{2}^{2}\right) \sin 2 \theta}{2 v_{s} v} \\
\mu_{H}^{2} & =-\frac{1}{4}\left(m_{1}^{2}+m_{2}^{2}\right)+\frac{1}{4 v}\left(m_{2}^{2}-m_{1}^{2}\right)\left(v \cos 2 \theta+v_{s} \sin 2 \theta\right), \\
\mu_{S}^{2} & =-\frac{1}{4}\left(m_{1}^{2}+m_{2}^{2}\right)+\frac{1}{6} m_{\chi}^{2}+\frac{1}{4 v_{s}}\left(m_{1}^{2}-m_{2}^{2}\right)\left(v_{s} \cos 2 \theta-v \sin 2 \theta\right), \\
\mu_{3} & =-\frac{2 \sqrt{2}}{9} \frac{m_{\chi}^{2}}{v_{s}}
\end{aligned}
$$



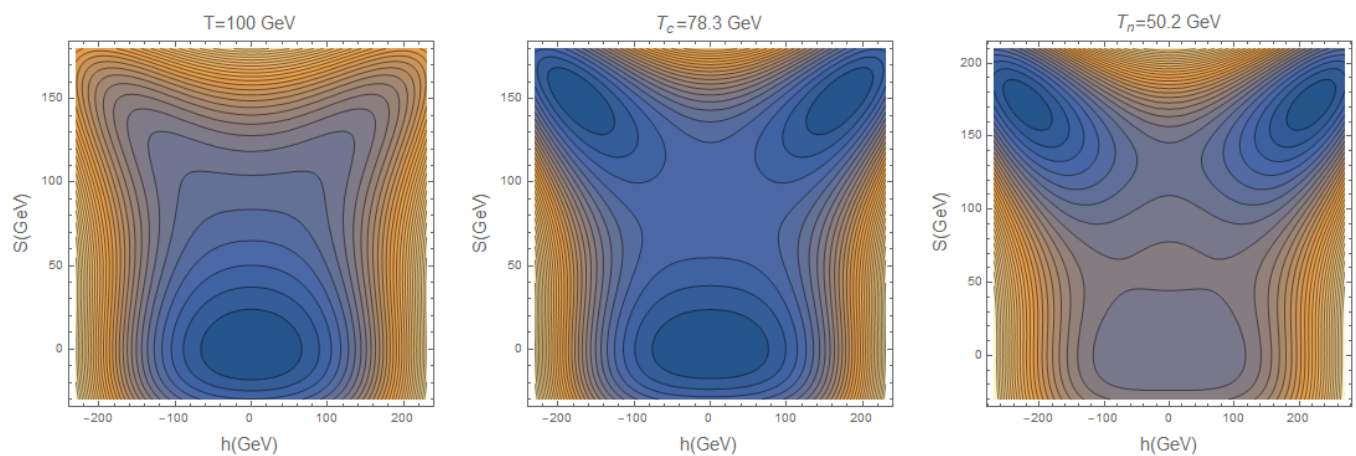

Figure 1. We plot the isocontours of the finite temperature potential to show the evolution of the vacuum structure as the temperature drops (with $\mathrm{BM}_{1}$ of the table 1 ). The left panel shows that both electroweak and $\mathbb{Z}_{3}$ symmetries are restored and the vacuum locates at $O(0,0)$ with a high temperature $T=100 \mathrm{GeV}$; the middle panel shows that the electroweak and $\mathbb{Z}_{3}$ broken minimum is degenerate with the minimum located at $O(0,0)$ at $T=T_{c}$; the right panel shows that the vacuum transits from $O(0,0)$ to the one locating at $B(h, S)$ at $T=T_{n}$, where we have both electroweak and $\mathbb{Z}_{3}$ symmetry broken.

\section{Electroweak phase transition}

In this section, we first investigate the phase transition dynamic relevant for the domain wall formation. After that, we evaluate the strongly first-order EWPT condition given by BNPC.

\subsection{Phase transition dynamic}

Due to the model belongs to the tree-level phase transition model class with the tree-level cubic term (here it is the $\mu_{3}$ term) providing a potential barrier for first-order EWPT [18], we utilize the gauge invariant approach $[12,13,32-36]$ which can provide a transparent physical intuition [37]. ${ }^{2}$ The finite temperature potential adopted for the study of the phase transition is given by

$$
V_{T}=\frac{\left(\mu_{H}^{2}+c_{h T}\right) h^{2}}{2}+\frac{\left(\mu_{s}^{2}+c_{s T}\right) s^{2}}{2}+\frac{\mu_{3} s^{3}}{2 \sqrt{2}}+\frac{\lambda_{H} h^{4}}{4}+\frac{\lambda_{S} s^{4}}{4}+\frac{\lambda_{\mathrm{SH}} h^{2} s^{2}}{4},
$$

with the finite temperature corrections are calculated as

$$
\begin{aligned}
c_{h T} & =\frac{1}{48} T^{2}\left(9 g^{2}+3 g^{2}+4\left(3 y_{t}^{2}+6 \lambda_{H}+\lambda_{\mathrm{SH}}\right)\right), \\
c_{s T} & =\frac{1}{6} T^{2}\left(2 \lambda_{S}+\lambda_{S H}\right) .
\end{aligned}
$$

Depending on the vacuum structure at the zero temperature, there are two different phase transition patterns, which are one-step phase transition $(O(0,0) \rightarrow B(h, s))$ and twostep phase transition $(O(0,0) \rightarrow A(h, 0)($ or $C(0, s)) \rightarrow B(h, s))$, see appendix. A for details.

\footnotetext{
${ }^{2}$ We note that additional strongly first-order EWPT parameter spaces may open up if one take the conventional approach to study the phase transition by including four ingredients [39]: the tree-level potential, the Coleman-Weinberg potential [38], the finite temperature corrections, and the daisy resummation.
} 
In this study, we focus on the one-step phase transition type. When the temperature of the Universe drops to the critical temperature, one have two degenerate vacua $O(0,0)$ and $B(h, s)$ with a potential barrier structure. Where, one has

$$
\begin{aligned}
V_{T}\left(0,0, T_{C}\right)=V_{T}\left(h_{B}, s_{B}, T_{C}\right), & \left.\frac{d V_{T}\left(h, s, T_{C}\right)}{d h}\right|_{h=h_{B}, s=s_{B}}=0, \\
& \left.\frac{d V_{T}\left(h, s, T_{C}\right)}{d s}\right|_{h=h_{B}, s=s_{B}}=0 .
\end{aligned}
$$

Through which, critical temperature and critical classical field value can be obtained. We note that, to ensure the appearance of the two degenerate vacua, the following constrains should be satisfied (a positive determination of the finite temperature Hessian matrix): $M_{1} P_{1}-N_{1}^{2}>0, M_{1}>0$, with

$$
\begin{aligned}
& \left.\frac{d^{2} V_{T}\left(h, s, T_{C}\right)}{d h^{2}}\right|_{h=h_{B}, s=s_{B}} \equiv M_{1},\left.\quad \frac{d^{2} V_{T}\left(h, s, T_{C}\right)}{d h d s}\right|_{h=h_{B}, s=s_{B}} \equiv N_{1}, \\
& \left.\frac{d^{2} V_{T}\left(h, s, T_{C}\right)}{d s^{2}}\right|_{h=h_{B}, s=s_{B}} \equiv P_{1} .
\end{aligned}
$$

We first select parameter points fulfilling the condition of $v_{c} / T_{c}>1$ at the critical temperature $T_{c}$ by using the above methodology, and then study if the bubble nucleation can occur and if the phase transition can complete with the public code CosmoTransitions [40]. In figure 1, we show a sample of the one-step phase transition process $(O(0,0) \rightarrow B(h, s))$. As the Universe cools down, a second minimum $B(h, s)$ develops at temperature $T_{n}$, which indicates the breakdown of the $\mathbb{Z}_{3}$ symmetry and the electroweak symmetry. Technically, we study the phase transition dynamics with free parameters falling into the ranges: $m_{\chi} \in[25,1000] \mathrm{GeV}, m_{2} \in[200,1000] \mathrm{GeV}, v_{s} \in[0,500] \mathrm{GeV}$, and $|\sin \theta| \leq 0.37$ considering the mixing angle is constrained by the current measurements of the Higgs couplings at the LHC searches [41]. For this study, the potential should be bounded from below with the following conditions,

$$
\lambda_{H}>0, \quad \lambda_{S}>0, \quad \lambda_{\mathrm{SH}}+2 \sqrt{\lambda_{H} \lambda_{S}}>0 .
$$

The unitarity constraints are,

$$
\begin{gathered}
\left|\lambda_{H}\right| \leqslant 4 \pi, \quad\left|\lambda_{S}\right| \leqslant 4 \pi, \quad\left|\lambda_{\mathrm{SH}}\right| \leqslant 8 \pi, \\
\left|3 \lambda_{H}+2 \lambda_{S} \pm \sqrt{9 \lambda_{H}^{2}-12 \lambda_{H} \lambda_{S}+4 \lambda_{S}^{2}+2 \lambda_{\mathrm{SH}}^{2}}\right| \leqslant 8 \pi .
\end{gathered}
$$

In figure 2, we show the distribution of EWPT points in various model parameters. The left (middle) panel indicates that the first-order EWPT can be obtained at a low value of $m_{\chi}$ and a large $m_{h_{2}}$, together with a large mixing angle $\theta$ (a small $v_{s}$ ). The right panel depicts that a stronger phase transition can be obtained with a larger pseudo-scalar mass $m_{\chi}$ and a larger mixing angle $\theta$. These plots demonstrate a clear relation between the phase transition dynamics and the $m_{\chi}$ (which is proportional to $\mu_{3}$ as shown in eq. (2.10)), which reflects that the tree-level cubic term (the $\mu_{3}$ term) trigger the phase transition as stated at the beginning of this section. 

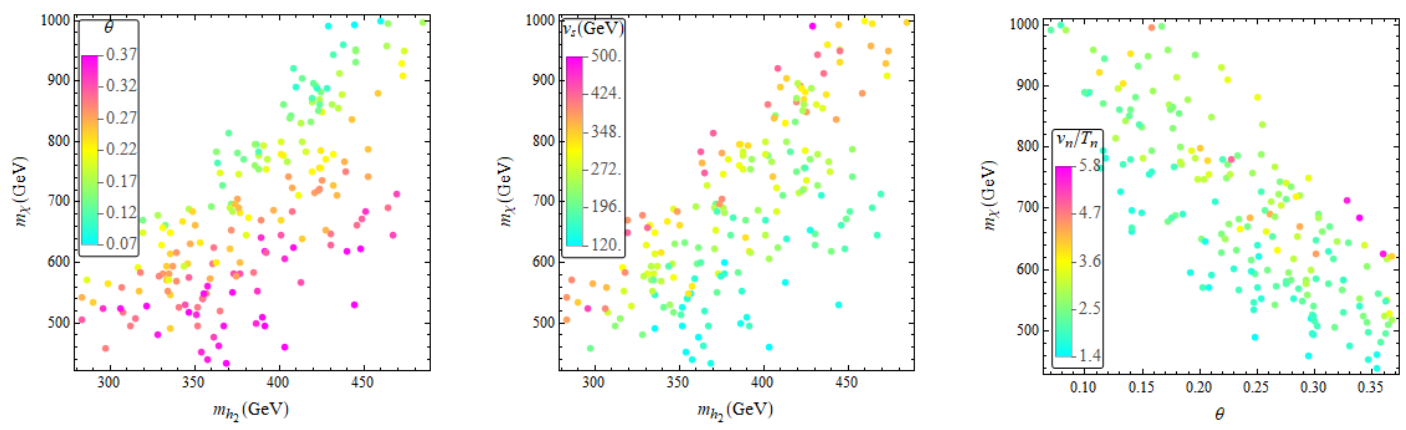

Figure 2. Left: the relation among $\theta, m_{\chi}$, and $m_{h_{2}}$ for EWPT points; middle: the relation among $v_{s}, m_{\chi}$, and $m_{h_{2}}$ for EWPT points; right: the phase transition strength $v_{n} / T_{n}$ as a function of $m_{\chi}$ and $\theta$.

\subsection{BNPC and the strongly first-order EWPT condition}

In this section, we study the strongly first-order EWPT condition through estimating the BNPC [42]. We first calculate the electroweak sphaleron energy $E_{\mathrm{sph}}(T)$ at the phase transition temperature, and then check the relation between the phase transition strength $v(T) / T$ and the following quantity as suggested in refs. [43, 44],

$$
P T_{\mathrm{sph}} \equiv \frac{E_{\mathrm{sph}}(T)}{T}-7 \ln \frac{v(T)}{T}+\ln \frac{T}{100 \mathrm{GeV}} .
$$

With the quantity $P T_{\mathrm{sph}}$ at hand, we check if the $\mathrm{BNPC}$ can meet as required by the successful baryon asymmetry generation within the electroweak baryogengesis paradigm [3, 42]. In particular, we check if the EWPT points satisfy the following condition [45]:

$$
P T_{\mathrm{sph}}>(35.9-42.8)
$$

The numerical range here mostly come from the uncertainty of the fluctuation determinant $\kappa=\left(10^{-4}-10^{-1}\right)[46]$, which is estimated to be comparable with the uncertainty in the lattice simulation of the sphaleron rate at the Standard Model electroweak cross-over [5, 45]. The numerical results are shown in figure 3, which shows that all the EWPT points satisfy the BNPC, and the sphaleron energy $E_{\mathrm{sph}}\left(T_{n}\right)$ and $P T_{\mathrm{sph}}$ increase with the growing of the phase transition strength $v_{n} / T_{n}$. Therefore, the washout of the baryon asymmetry can be avoided. For uncertainty from the duration of the EWPT and bubble nucleation involved in the evaluation of the BNPC, we refer to ref. [42].

\section{Gravitational wave}

Because we have the electroweak symmetry and $\mathbb{Z}_{3}$ symmetry broken simultaneously at the phase transition, we expect two sources of the gravitational wave radiation produced at the early Universe. In this section, we study the stochastic gravitational waves from the strongly first-order EWPT and the domain wall decay after that. 


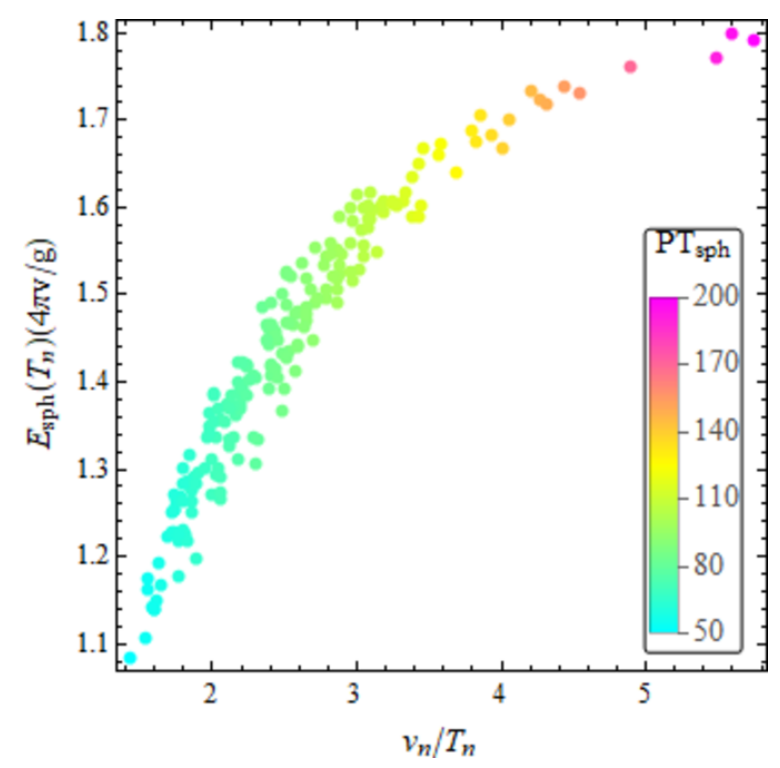

Figure 3. The $P T_{\mathrm{sph}}$ as a function of the electroweak sphaleron energy at nucleation temperature and the phase transition strength $v_{n} / T_{n}$.

\subsection{Gravitational waves from the first-order EWPT}

One crucial parameter for the calculation of the gravitational wave is $\alpha$, which is the energy budget of the first-order EWPT normalized by the radiative energy, and is defined as

$$
\alpha=\frac{\Delta \rho}{\rho_{R}} .
$$

Here, $\rho_{R}=\pi^{2} g_{\star} T_{\star}^{4} / 30$ is radiation energy of the bath or the plasma background, and $\Delta \rho$ is the latent heat from the phase transition to the energy density of the radiation bath or the plasma background. We take $T_{\star} \approx T_{n}$. There is another parameter $\beta$ which characterizes the inverse time duration of the phase transition, and is defined as

$$
\frac{\beta}{H_{n}}=\left.T \frac{d\left(S_{3}(T) / T\right)}{d T}\right|_{T=T_{n}}
$$

Both the two parameters can be calculated after the solution of the bounce configuration (the bounce configuration of the field connects the electroweak broken vacuum (true vacuum, $\mathbb{Z}_{3}$ is broken) and the electroweak preserving vacuum (false vacuum, $\mathbb{Z}_{3}$ is preserving)) of the nucleated bubble, which is obtained by extremizing,

$$
S_{3}(T)=\int 4 \pi r^{2} d r\left[\frac{1}{2}\left(\frac{d \phi_{b}}{d r}\right)^{2}+V\left(\phi_{b}, T\right)\right]
$$

through solving the equation of motion for the field $\phi_{b}$ (it is subspace of $h$ and $s$ in this study), with the boundary conditions

$$
\lim _{r \rightarrow \infty} \phi_{b}=0,\left.\quad \frac{d \phi_{b}}{d r}\right|_{r=0}=0 .
$$



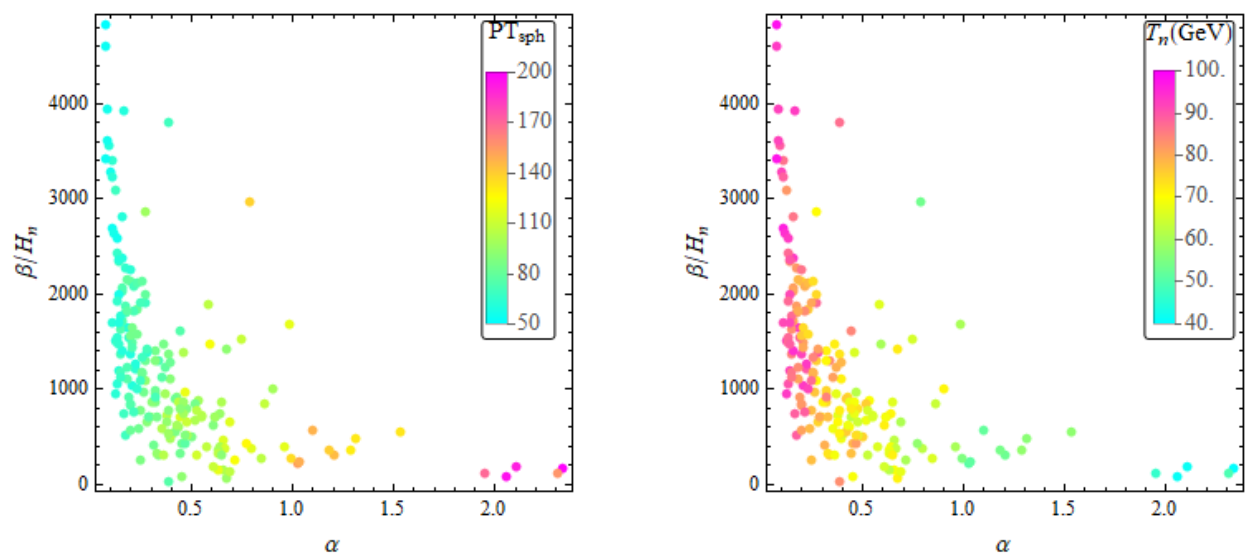

Figure 4. Left: we plot the relation between $\alpha$ and $\beta / H_{n}$ with the $P T_{\mathrm{sph}}$ as color-code; right: we show gravitational wave parameters $\beta / H_{n}$ and $\alpha$, with nucleation temperature $T_{n}$ as color-code.

The phase transition completes at the nucleation temperature when the thermal tunneling probability for bubble nucleation per horizon volume and per horizon time is of order unity [47-49]:

$$
\Gamma \approx A(T) e^{-S_{3} / T} \sim 1
$$

Before going to the study of gravitational wave, we first present the relation among the quantity of $P T_{\mathrm{sph}}$ and the two crucial parameters for gravitational wave $\left(\alpha, \beta / H_{n}\right)$ in the left panel of the figure 4 . Which shows that a smaller $\beta / H_{n}$ (i.e., a long phase transition duration time) and a larger $\alpha$ (a larger phase transition strength) corresponds to a larger $P T_{\mathrm{sph}}$, where the BNPC can be satisfied much better. In the right panel of the figure 4, we present the relation among the nucleation temperature $T_{n}$ and the two parameters for gravitational wave $\left(\alpha, \beta / H_{n}\right)$. Which depicts that a large $\alpha$ along with a small $\beta / H_{n}$ can be reached for a small $T_{n}$, where one can expect a strong gravitational wave [13]. Indeed, the two plots also demonstrate that a lower bubble nucleation temperature $T_{n}$ leads to a larger $P T_{\mathrm{sph}}$.

The gravitational waves from the first-order EWPT mainly come from sound waves and MHD turbulence, with the total energy being given by [50]

$$
\Omega_{\mathrm{GW}} h^{2}(f) \approx \Omega h_{\mathrm{sW}}^{2}(f)+\Omega h_{\mathrm{turb}}^{2}(f) .
$$

Here, we consider detonation bubble, and take the bubble wall velocity $v_{b}$ as a function of $\alpha[51],{ }^{3}$

$$
v_{b}=\frac{1 / \sqrt{3}+\sqrt{\alpha^{2}+2 \alpha / 3}}{1+\alpha} .
$$

The peak frequency of the sound wave locates at $[52,53]$

$$
f_{\mathrm{sw}}=1.9 \times 10^{-5} \frac{\beta}{H} \frac{1}{v_{b}} \frac{T_{*}}{100}\left(\frac{g_{*}}{100}\right)^{\frac{1}{6}} \mathrm{~Hz},
$$

\footnotetext{
${ }^{3}$ We note that to compatible with EWBG, the wall velocity here can be obtained as a function of $\alpha$. [9, 12, 13, 73] after taking into account Hydrodynamics.
} 
with the energy density being given by

$$
\Omega h_{\mathrm{sw}}^{2}(f)=2.65 \times 10^{-6}\left(\frac{\beta}{H}\right)^{-1}\left(\frac{\kappa \alpha}{1+\alpha}\right)^{2}\left(\frac{g_{*}}{100}\right)^{-\frac{1}{3}} v_{b}\left(\frac{f}{f_{\mathrm{sw}}}\right)^{3}\left(\frac{7}{4+3\left(f / f_{\mathrm{sw}}\right)^{2}}\right)^{7 / 2} .
$$

Here, the $\kappa$ describes the fraction of the latent heat transferred into the kinetic energy of plasma, we obtain the value by considering the the hydrodynamic analysis [54]. The MHD turbulence in the plasma is the second important source of the gravitational wave signals from phase transition, the peak frequency locates at [55]

$$
f_{\text {turb }}=2.7 \times 10^{-5} \frac{\beta}{H} \frac{1}{v_{b}} \frac{T_{*}}{100}\left(\frac{g_{*}}{100}\right)^{\frac{1}{6}} \mathrm{~Hz}
$$

and the energy density is

$$
\Omega h_{\text {turb }}^{2}(f)=3.35 \times 10^{-4}\left(\frac{\beta}{H}\right)^{-1}\left(\frac{\epsilon \kappa \alpha}{1+\alpha}\right)^{\frac{3}{2}}\left(\frac{g_{*}}{100}\right)^{-\frac{1}{3}} v_{b} \frac{\left(f / f_{\text {turb }}\right)^{3}\left(1+f / f_{\text {turb }}\right)^{-\frac{11}{3}}}{\left[1+8 \pi f a_{0} /\left(a_{*} H_{*}\right)\right]}
$$

where the efficiency factor $\epsilon \approx 0.05$, and the precent Hubble parameter is

$$
h_{*}=\left(1.65 \times 10^{-5} \mathrm{~Hz}\right)\left(\frac{T_{*}}{100 \mathrm{GeV}}\right)\left(\frac{g_{*}}{100}\right)^{1 / 6} .
$$

\subsection{Gravitational waves from domain wall decay}

To get domain wall solution formed after the phase transition [56], we first introduce the phase of the singlet as $S=v_{s} e^{i \phi}$, and obtain the potential of $\phi$ as:

$$
V=\frac{\mu_{H}^{2}}{2} v^{2}+\frac{\lambda_{H}}{4} v^{4}+\frac{\mu_{S}^{2}}{2} v_{s}^{2}+\frac{\lambda_{S}}{4} v_{s}^{4}+\frac{\lambda_{\mathrm{SH}}}{4} v_{s}^{2} v^{2}+\frac{\mu_{3}}{2 \sqrt{2}} v_{s}^{3} \cos (3 \phi) .
$$

With $\eta^{2}=v_{s}^{2} / 2$, the kinetic term of $\phi$ can be obtained as,

$$
\mathcal{L}_{\text {kinetic }}(\phi)=\eta^{2}\left(\partial_{\mu} \phi\right)\left(\partial^{\mu} \phi\right) \text {. }
$$

The field equation,

$$
\partial_{\mu} \frac{\partial \mathcal{L}_{\text {kinetic }}}{\partial_{\mu}(\partial \phi)}+\frac{\partial V}{\partial \phi}=0
$$

yields

$$
\frac{\mathrm{d}^{2} \phi}{\mathrm{d} z^{2}}-\frac{1}{3 B^{2}} \sin (3 \phi)=0
$$

with

$$
\frac{1}{B^{2}}=-\frac{9}{4} \mu_{3} v_{s}^{2}, \quad \phi=\frac{4}{3} \arctan \left(e^{\frac{z}{B}}\right) .
$$

From which, we can consider a planar domain wall orthogonal to the z-axis [57], i.e., $\phi(z)$. The domain wall tension is estimated as,

$$
\sigma=\int d z \rho_{\text {wall }}(z)=\int\left(\left|\frac{d S}{d z}\right|^{2}+V\left(\frac{S(z)}{\sqrt{2}}, \frac{v}{\sqrt{2}}\right)-V\left(\frac{v_{s}}{\sqrt{2}}, \frac{v}{\sqrt{2}}\right)\right) d z .
$$


The same as the previous study of gravitational waves at EWPT, we assume the gravitational radiation produced in the radiation dominated era. After the formation of the domain wall after the EWPT, one have the domain wall decay. With the peak frequency is given by the Hubble parameter at the decay time [58]:

$$
f^{\mathrm{dw}}\left(t_{0}\right)_{\text {peak }}=\frac{a\left(t_{\mathrm{dec}}\right)}{a\left(t_{0}\right)} H\left(t_{\mathrm{dec}}\right) \simeq 3.99 \times 10^{-9} \mathrm{~Hz}^{-1 / 2}\left(\frac{1 \mathrm{TeV}^{3}}{\sigma_{\text {wall }}}\right)^{1 / 2}\left(\frac{\Delta V}{1 \mathrm{MeV}^{4}}\right)^{1 / 2}
$$

and peak amplitude of the gravitational waves at the present time $t_{0}$ is estimated as $[58,59]$

$$
\Omega_{\mathrm{GW}}^{\mathrm{dw}} h^{2}\left(t_{0}\right)_{\mathrm{peak}} \simeq 5.20 \times 10^{-20} \times \tilde{\epsilon}_{\mathrm{gw}} \mathcal{A}^{4}\left(\frac{10.75}{g_{*}}\right)^{1 / 3}\left(\frac{\sigma_{\mathrm{wall}}}{1 \mathrm{TeV}^{3}}\right)^{4}\left(\frac{1 \mathrm{MeV}^{4}}{\Delta V}\right)^{2} .
$$

Requiring the domain wall decay before they overclose Universe results in,

$$
\sigma_{\text {wall }}<2.93 \times 10^{4} \mathrm{TeV}^{3} \mathcal{A}^{-1}\left(\frac{0.1 \mathrm{sec}}{t_{\mathrm{dec}}}\right) .
$$

The bias term $\Delta V$ in eqs. (4.19), (4.20) is introduced to explicitly break the $\mathbb{Z}_{3}$ symmetry, which determines the decay time of the domain wall,

$$
t_{\mathrm{dec}} \approx \mathcal{A} \sigma_{\mathrm{wall}} /(\Delta V)
$$

Requiring the domain wall decay before the BBN with $t_{\text {dec }} \leq 0.01$ sec $[60,61]$, one has a lower limit on the magnitude of the bias term,

$$
\Delta V \gtrsim 6.6 \times 10^{-2} \mathrm{MeV}^{4} \mathcal{A}\left(\frac{\sigma_{\text {wall }}}{1 \mathrm{TeV}^{3}}\right) .
$$

We note that the magnitude of the bias term should be much less than that of the potential around the core of domain walls $(\Delta V \ll V)$, such that the discrete $\mathbb{Z}_{3}$-symmetry holds approximately and not affect the phase transition dynamics. In this study, we take the area parameter $\mathcal{A}=1.2$ for $\mathbb{Z}_{3}$ symmetry as in ref. [59], the efficiency parameter $\tilde{\epsilon}_{\mathrm{gw}}=0.7$ [58], and the degree of freedom at the domain wall decay time $g_{*}=10.75$ [59]. The whole spectrum of the gravitational wave can be obtained after considering the slope of spectrum $\Omega_{\mathrm{GW}}^{\mathrm{dw}} h^{2} \propto f^{3}$ when $f<s f_{\text {peak }}$, and $\Omega_{\mathrm{GW}}^{\mathrm{dw}} h^{2} \propto f^{-1}$ when $f \geqslant f_{\text {peak }}$ as estimated in ref. [58].

Eq. (4.19) and eq. (4.20) indicate that $f_{\text {peak }}^{\mathrm{dw}}$ is proportional to $\left(\frac{\Delta V}{\sigma_{\text {wall }}}\right)^{1 / 2}$ and $\Omega_{\mathrm{GW}}^{\mathrm{dw}} h^{2}$ is proportional to $\sigma_{\text {wall }}^{4}$. In order to evaluate the detectability of the gravitational waves from the domain wall after the strongly first-order EWPT, we fix $f_{\text {peak }}^{\mathrm{dw}} \approx 2 \times 10^{-9} \mathrm{~Hz}$ at the sensitivity frequency of PPTA [29] and get $\Delta V$ from the eq. (4.19) with $\sigma_{\text {wall }}$ been calculated using the strongly first-order EWPT points. As shown in figure 5, with increase of $\sigma_{\text {wall }}$ and $\Delta V, \Omega_{\mathrm{GW}}^{\mathrm{dw}} h^{2}$ can reach to $3.56 \times 10^{-18}$, which is far beyond the sensitivity of the current PTA and the future SKA [31]. Ref. [62] shows that a higher magnitude of the gravitational wave spectrum from the domain wall decay requires a large surface mass density of domain walls, which cannot be realized in the strongly first-order EWPT parameter spaces in this study. 


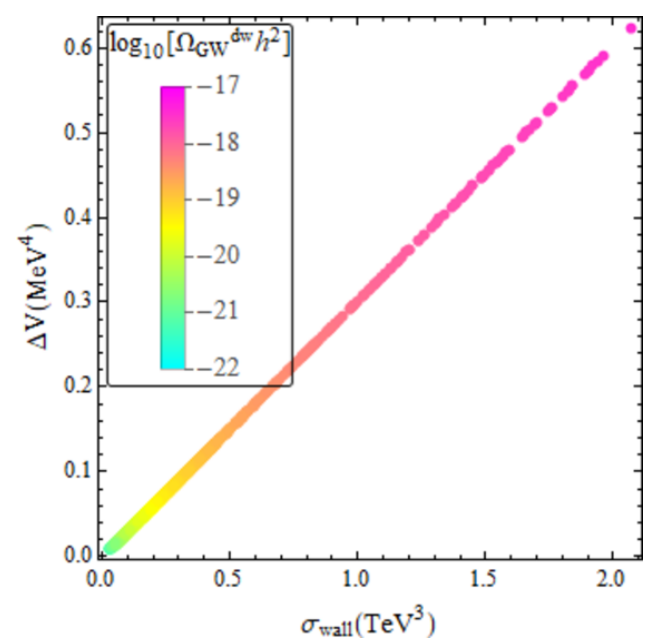

Figure 5. We show the relation between the surface mass density $\sigma_{\text {wall }}$ and the bias term $\Delta V$, with the $\Omega_{\mathrm{GW}}^{\mathrm{dw}} h^{2}$ as the color-code.

\begin{tabular}{|cccccccc|}
\hline & $m_{\chi}(\mathrm{GeV})$ & $m_{h_{2}}(\mathrm{GeV})$ & $v_{s}(\mathrm{GeV})$ & $\theta$ & $T_{n}(\mathrm{GeV})$ & $\beta / H_{n}$ & $\alpha$ \\
$B M_{1}$ & 625.08 & 361.31 & 184.10 & 0.30 & 50.16 & 219.62 & 1.02 \\
\hline$B M_{2}$ & 814.81 & 370.24 & 243.05 & 0.13 & 69.46 & 152.41 & 0.66 \\
\hline
\end{tabular}

Table 1. Benchmarks in the figure 6.

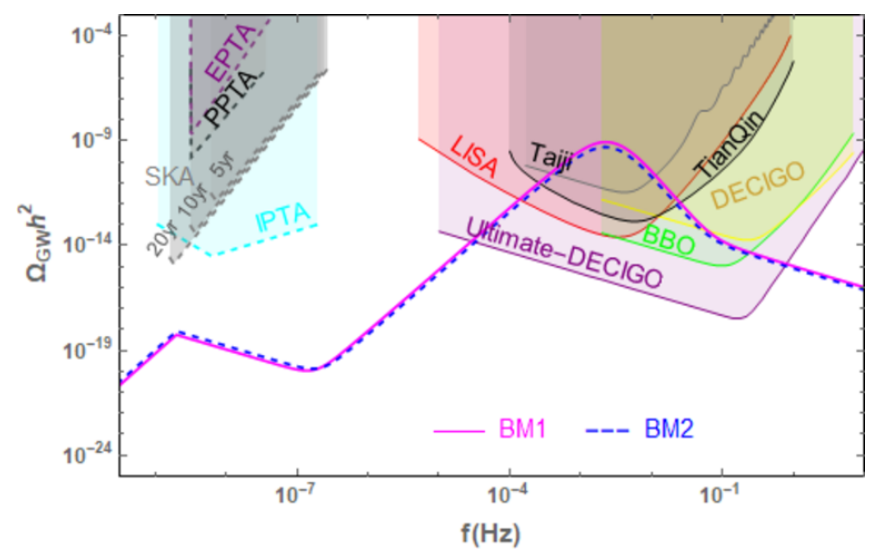

Figure 6. Gravitational wave signals from the strongly first-order EWPT and domain wall decay.

\subsection{Gravitational waves from strongly first-order EWPT and domain wall}

We present the gravitational waves radiation from the strongly first-order EWPT and domain wall decay in figure 6 after summing the two contributions. The gravitational waves from the strongly first-order EWPT is dominant in the higher frequency, and domain wall decay dominates the gravitational wave spectrum of the low frequency. The higher peak of the gravitational wave spectrum locates around $f_{\text {peak }} \sim 10^{-3}-10^{-2} \mathrm{~Hz}$, which can be probed by the projected space-based interferometers, such as: LISA [63], BBO [64], 
DECIGO (Ultimate-DECIGO) [65, 66], TianQin [67] and Taiji [68] programs. While, the gravitational wave spectrum locating around the lower peak from domain wall decay is too weak to be probed by Pulsar Timing Arrays and Square Kilometer Array.

\section{Conclusion and discussion}

With the $\mathbb{Z}_{3}$ symmetric complex singlet scalar model, we study the possibility to achieve a one-step strongly first-order EWPT after considering the baryon number preservation criterion. After that, we study the gravitational wave prediction from the strongly first-order EWPT with domain wall decay, a two-peak shape spectrum is found. The peak of the predicted gravitational wave from the domain wall decay locates around $f_{\text {peak }}^{\mathrm{dw}} \sim \mathcal{O}\left(10^{-9}\right) H z$, and the amplitude of the spectrum is beyond the sensitivity of EPTA, PPTA, IPTA, and SKA. The peak of the predicted gravitational wave spectrum from the phase transition locates at around $f_{\text {peak }}^{\text {pt }} \sim \mathcal{O}\left(10^{-3}-10^{-2}\right) H z$, and its strength falls into the capability of the projected space-base interferometers, such as: LISA, BBO, DECIGO, UDECIGO, TianQin and Taiji.

\section{Acknowledgments}

This work is supported by the National Natural Science Foundation of China under grant No.11605016 and No.11647307, and the Fundamental Research Funds for the Central Universities of China (No. 2019CDXYWL0029). We are grateful to Tanmay Vachaspati, Alexander Vilenkin, Ken'ichi Saikawa, Salah Nasri, Michael J. Ramsey-Musolf, and HuaiKe Guo for helpful communications and discussions.

\section{A Vacuum structures and phase transition types}

As shown in figure 7, there are totally four possible minima locating at $O(0,0), A(0, s)$, $B(h, s)$, and $C(h, 0)$.

$$
\begin{array}{lll}
\text { O point : } & h \rightarrow 0, & S \rightarrow 0, \\
\text { A point }: & h \rightarrow 0, & S \rightarrow \frac{9 \mu_{3}^{2}-16 \lambda_{S} \mu_{S}^{2}-3 \sqrt{9 \mu_{3}^{4}-32 \lambda_{S} \mu_{3}^{2} \mu_{S}^{2}}}{16 \lambda_{S}^{2}}, \\
\text { B point : } & h \rightarrow h_{B}, & S \rightarrow S_{B}, \\
\text { C point : } & h \rightarrow \sqrt{\frac{-\mu_{H}^{2}}{\lambda_{H}}}, & S \rightarrow 0 .
\end{array}
$$

Therefore, three types phase transition process could happen: one-step phase transition $(O \rightarrow B)$ (Blue), and two-step phase transition $(O \rightarrow A(C) \rightarrow B)$ (Magenta(Red)). The one-step phase transition points gather around the $\theta>0$ region. For parameter space with a negative $\theta$, the two-step phase transition $O \rightarrow C \rightarrow B$ could take place. Meanwhile, the two-step phase transition $(O \rightarrow A \rightarrow B)$ occurs with $\theta \geq 0.35$ that is not favored by the current LHC measurements. 

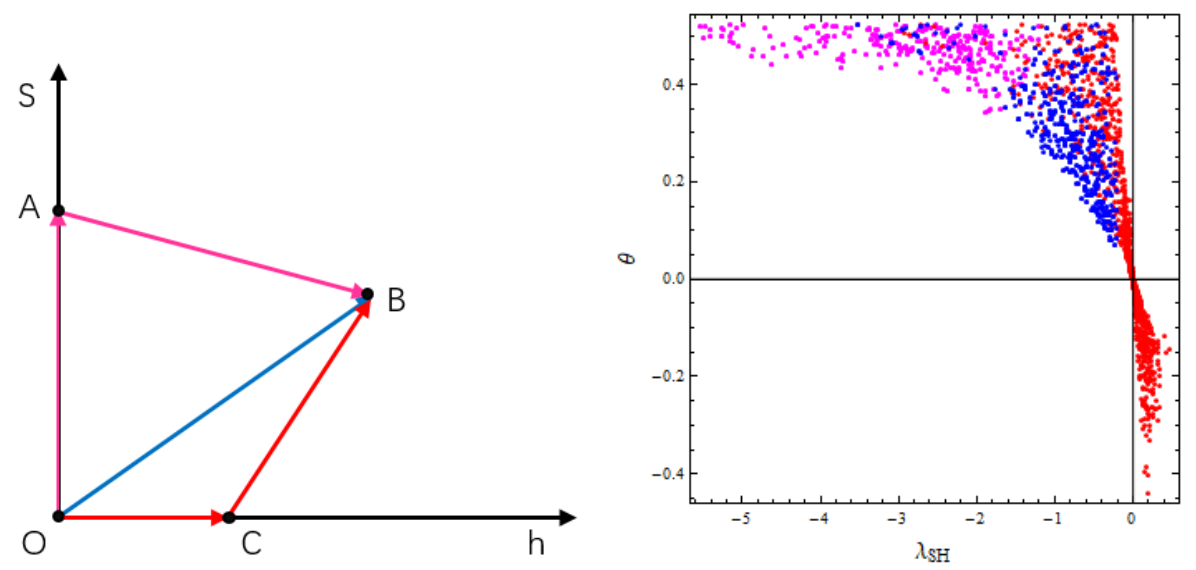

Figure 7. Left: the tree level potential vacuum structure of the $\mathbb{Z}_{3}$ symmetric complex singlet scalar model. Right: we show the three types phase transition points distribution in the $\theta-\lambda_{\text {SH }}$ plane.

\section{B Electroweak sphaleron}

To compute $E_{\mathrm{sph}}(T)$, we obtain the sphaleron solutions following a method suggested in refs. [69, 70]. Since $\mathrm{U}(1)_{Y}$ contributions are sufficiently small [71, 72], we employ the spherically symmetric ansatz. Specifically, the configuration of gauge, Higgs and singlet scalar fields are expressed as:

$$
\begin{aligned}
A_{i}(\mu, r, \theta, \phi) & =-\frac{i}{g} f(r) \partial_{i} U(\mu, \theta, \phi) U^{-1}(\mu, \theta, \phi), \\
H(\mu, r, \theta, \phi) & =\frac{v[T]}{\sqrt{2}}\left[(1-h(r))\left(\begin{array}{c}
0 \\
e^{-i \mu} \cos \mu
\end{array}\right)+h(r) U(\mu, \theta, \phi)\left(\begin{array}{l}
0 \\
1
\end{array}\right)\right], \\
S(\mu, r, \theta, \phi) & =\frac{v_{s}(T)}{\sqrt{2}} s(r) .
\end{aligned}
$$

where $A_{i}$ are $\mathrm{SU}(2)$ gauge fields, $A_{i}=\frac{1}{2} A_{i}^{a} \tau^{a}$, and $U(\mu, \theta, \phi)$ is defined as

$$
U(\mu, \theta, \phi)=\left(\begin{array}{cc}
e^{i \mu}(\cos \mu-i \sin \mu \cos \theta) & e^{i \phi} \sin \mu \sin \theta \\
-e^{-i \phi} \sin \mu \sin \theta & e^{-i \mu}(\cos \mu+i \sin \mu \cos \theta)
\end{array}\right) .
$$

The sphaleron energy in the finite temperature can be calculated as:

$$
\begin{aligned}
E_{\mathrm{sph}}(T)= & \frac{4 \pi \Omega[T]}{g} \int_{0}^{\infty} d \xi\left[4\left(\frac{d f}{d \xi}\right)^{2} s_{\mu}^{2}+\frac{8}{\xi^{2}} f^{2}(1-f)^{2} s_{\mu}^{4}+\frac{\xi^{2} v[T]^{2}}{2 \Omega[T]^{2}}\left(\frac{d h}{d \xi}\right)^{2} s_{\mu}^{2}\right. \\
& +\frac{\xi^{2} v_{s}[T]^{2}}{2 \Omega[T]^{2}}\left(\frac{d s}{d \xi}\right)^{2}+s_{\mu}^{2} \frac{v[T]^{2}}{\Omega[T]^{2}}\left((1-f)^{2} h^{2}-2 f h(1-f)(1-h) c_{\mu}^{2}+f^{2}(1-h)^{2} c_{\mu}^{2}\right) \\
& \left.+\frac{\xi^{2}}{g^{2} \Omega[T]^{4}} V_{\mathrm{eff}}[\mu, h, s, T]\right],
\end{aligned}
$$

when $\mu=\pi / 2$, where $\xi=g \Omega[T] r, V_{\text {eff }}[\mu, h, s, T]=V_{\text {potential }}[\mu, h, s, T]-|\Delta[T]|$, and $\Delta[T]$ is the cosmological constant energy density. The $\Delta[T]$ can be regarded as the minimal value 

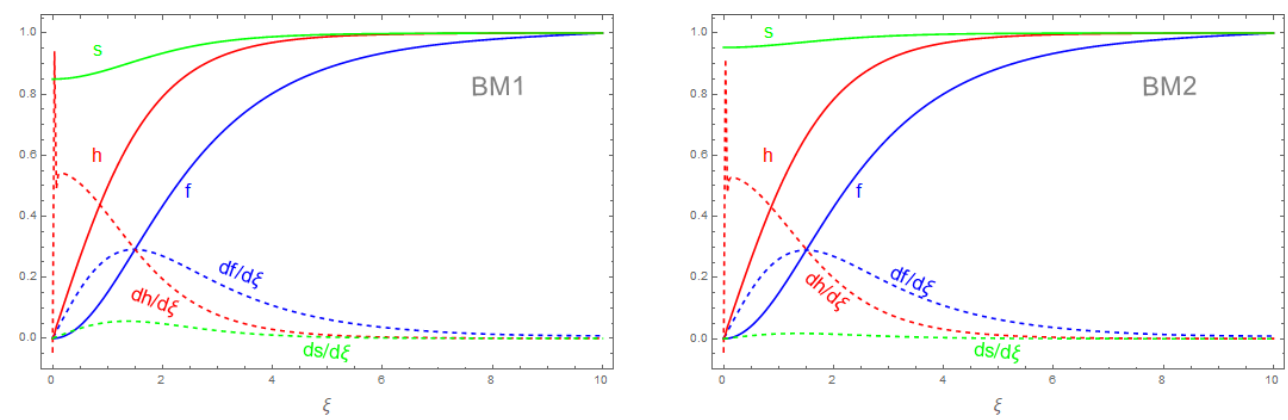

Figure 8. Numerically solved $\operatorname{Sphaleron}(\mu=\pi / 2)$ profiles of $\mathrm{f}, \mathrm{h}, \mathrm{s}$ and their derivatives as a function of the dimensionless quantity $\xi$ for the two benchmarks in table 1 .
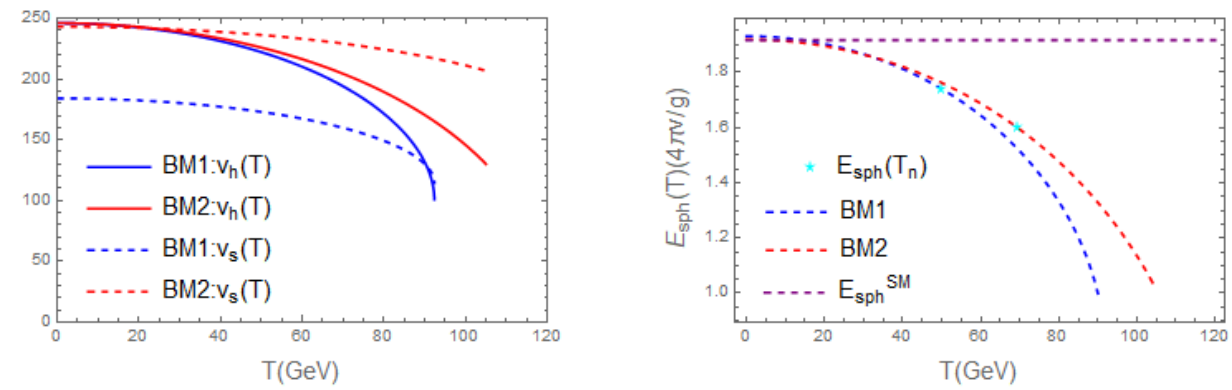

Figure 9. Left: finite temperature VEVs of h(blue) and s(red) versus $T$ for the two benchmark points given in table 1. Right: electroweak sphaleron energy as a function of temperature.

of the potential at temperature $T$. The parameter $\Omega[T]$ can take any nonvanishing value of mass dimension one (for example $v[T], v_{S}[T]$ or $\sqrt{v[T]^{2}+v_{S}[T]^{2}}$ ). From eq. (B.5), the equations of motion are found to be

$$
\begin{aligned}
\frac{d^{2} f}{d \xi^{2}} & =\frac{2}{\xi^{2}} f(1-f)(1-2 f) s_{\mu}^{2}+\frac{1}{4}\left(h^{2}(f-1)-h(1-h)(1-2 f) c_{\mu}^{2}+f(1-h)^{2} c_{\mu}^{2}\right), \\
\frac{d}{d \xi}\left(\xi^{2} \frac{d h}{d \xi}\right) & =2 h(1-f)^{2}-2 f(1-f)(1-2 h) c_{\mu}^{2}-2 f^{2}(1-h) c_{\mu}^{2}+\frac{\xi^{2}}{g^{2}} \frac{1}{v[T]^{2} \Omega[T]^{2}} \frac{\partial V_{\mathrm{eff}}}{\partial h} \\
\frac{d}{d \xi}\left(\xi^{2} \frac{d s}{d \xi}\right) & =\frac{\xi^{2}}{g^{2}} \frac{1}{v_{S}[T]^{2} \Omega[T]^{2}} \frac{\partial V_{\mathrm{eff}}}{\partial s} .
\end{aligned}
$$

The sphaleron solutions could be obtained with the boundary conditions:

$$
\begin{array}{lll}
\lim _{\xi \rightarrow 0} f(\xi)=0, & \lim _{\xi \rightarrow 0} h(\xi)=0, & \lim _{\xi \rightarrow 0} s^{\prime}(\xi)=0, \\
\lim _{\xi \rightarrow \infty} f(\xi)=1, & \lim _{\xi \rightarrow \infty} h(\xi)=1, & \lim _{\xi \rightarrow \infty} s(\xi)=1 .
\end{array}
$$

In figure 8, we demonstrate the profile of the Higgs field, SU(2) gauge field, singlet scalar field, and their derivative behaviors, respectively. In figure 9, we illustrate that the sphaleron energy $E_{\mathrm{sph}}(T)$ and VEVs of $h\left(v_{h}\right)$ and $s\left(v_{s}\right)$ decrease as the temperature drops for the two benchmark points in table 1 . The sphaleron energy $E_{\mathrm{sph}}(T)$ is highly sensitive to the VEV of $h$, as indicated in ref. [42]. 
Open Access. This article is distributed under the terms of the Creative Commons Attribution License (CC-BY 4.0), which permits any use, distribution and reproduction in any medium, provided the original author(s) and source are credited.

\section{References}

[1] LiGO Scientific, Virgo collaboration, Observation of gravitational waves from a binary black hole merger, Phys. Rev. Lett. 116 (2016) 061102 [arXiv: 1602.03837] [INSPIRE].

[2] LISA collaboration, Laser Interferometer Space Antenna, arXiv:1702.00786 [INSPIRE].

[3] D.E. Morrissey and M.J. Ramsey-Musolf, Electroweak baryogenesis, New J. Phys. 14 (2012) 125003 [arXiv: 1206.2942].

[4] C. Caprini et al., Detecting gravitational waves from cosmological phase transitions with LISA: an update, JCAP 03 (2020) 024 [arXiv:1910.13125] [INSPIRE].

[5] M. D'Onofrio, K. Rummukainen and A. Tranberg, Sphaleron rate in the minimal standard model, Phys. Rev. Lett. 113 (2014) 141602 [arXiv:1404.3565] [INSPIRE].

[6] A. Mazumdar and G. White, Review of cosmic phase transitions: their significance and experimental signatures, Rept. Prog. Phys. 82 (2019) 076901 [arXiv:1811.01948] [InSPIRE].

[7] N. Arkani-Hamed, T. Han, M. Mangano and L.-T. Wang, Physics opportunities of a $100 \mathrm{TeV}$ proton-proton collider, Phys. Rept. 652 (2016) 1 [arXiv:1511.06495] [INSPIRE].

[8] N. Chen, T. Li, Y. Wu and L. Bian, Discriminate the discrete symmetry through the future $e^{+} e^{-}$colliders and gravitational waves, arXiv:1911.05579 [INSPIRE].

[9] A. Alves et al., Di-Higgs production in the $4 b$ channel and gravitational wave complementarity, JHEP 03 (2020) 053 [arXiv: 1909.05268] [INSPIRE].

[10] K. Hashino et al., Selecting models of first-order phase transitions using the synergy between collider and gravitational-wave experiments, Phys. Rev. D 99 (2019) 075011 [arXiv: 1809.04994] [INSPIRE].

[11] K. Hashino, M. Kakizaki, S. Kanemura and T. Matsui, Synergy between measurements of gravitational waves and the triple-Higgs coupling in probing the first-order electroweak phase transition, Phys. Rev. D 94 (2016) 015005 [arXiv:1604.02069] [INSPIRE].

[12] L. Bian, H.-K. Guo, Y. Wu and R. Zhou, Gravitational wave and collider searches for electroweak symmetry breaking patterns, Phys. Rev. D 101 (2020) 035011 [arXiv: 1906.11664] [INSPIRE].

[13] A. Alves et al., Collider and gravitational wave complementarity in exploring the singlet extension of the standard model, JHEP 04 (2019) 052 [arXiv:1812.09333] [INSPIRE].

[14] T.W.B. Kibble, Topology of cosmic domains and strings, J. Phys. A 9 (1976) 1387 [inSPIRE].

[15] A. Vilenkin, Gravitational field of vacuum domain walls and strings, Phys. Rev. D 23 (1981) 852 [INSPIRE].

[16] G.B. Gelmini, M. Gleiser and E.W. Kolb, Cosmology of biased discrete symmetry breaking, Phys. Rev. D 39 (1989) 1558 [inSPIRE].

[17] S.E. Larsson, S. Sarkar and P.L. White, Evading the cosmological domain wall problem, Phys. Rev. D 55 (1997) 5129 [hep-ph/9608319] [inSPIRE].

[18] D.J.H. Chung, A.J. Long and L.-T. Wang, 125 GeV Higgs boson and electroweak phase transition model classes, Phys. Rev. D 87 (2013) 023509 [arXiv:1209.1819] [INSPIRE]. 
[19] E. Ma, $Z_{3}$ dark matter and two-loop neutrino mass, Phys. Lett. B 662 (2008) 49 [arXiv:0708.3371] [INSPIRE].

[20] G. Bélanger, K. Kannike, A. Pukhov and M. Raidal, $Z_{3}$ scalar singlet dark matter, JCAP 01 (2013) 022 [arXiv:1211.1014] [INSPIRE].

[21] Y. Cai and A. Spray, Low-temperature enhancement of semi-annihilation and the AMS-02 positron anomaly, JHEP 10 (2018) 075 [arXiv:1807.00832] [INSPIRE].

[22] G. Arcadi, F.S. Queiroz and C. Siqueira, The semi-Hooperon: gamma-ray and anti-proton excesses in the Galactic Center, Phys. Lett. B 775 (2017) 196 [arXiv:1706.02336] [INSPIRE].

[23] A. Hektor, A. Hryczuk and K. Kannike, Improved bounds on $\mathbb{Z}_{3}$ singlet dark matter, JHEP 03 (2019) 204 [arXiv: 1901.08074] [INSPIRE].

[24] Z. Kang, P. Ko and T. Matsui, Strong first order EWPT \& strong gravitational waves in $Z_{3}$-symmetric singlet scalar extension, JHEP 02 (2018) 115 [arXiv:1706.09721] [INSPIRE].

[25] K. Kannike, K. Loos and M. Raidal, Gravitational wave signals of pseudo-Goldstone dark matter in the $Z_{3}$ complex singlet model, Phys. Rev. D 101 (2020) 035001 [arXiv: 1907.13136] [INSPIRE].

[26] C.-W. Chiang and B.-Q. Lu, First-order electroweak phase transition in a complex singlet model with $Z_{3}$ symmetry, arXiv:1912.12634 [INSPIRE].

[27] P.S.B. Dev, F. Ferrer, Y. Zhang and Y. Zhang, Gravitational waves from first-order phase transition in a simple axion-like particle model, JCAP 11 (2019) 006 [arXiv: 1905.00891] [INSPIRE].

[28] G. Desvignes et al., High-precision timing of 42 millisecond pulsars with the European Pulsar Timing Array, Mon. Not. Roy. Astron. Soc. 458 (2016) 3341 [arXiv:1602.08511] [InSPIRE].

[29] G. Hobbs, The parkes pulsar timing array, Class. Quant. Grav. 30 (2013) 224007 [arXiv:1307.2629] [INSPIRE].

[30] J.P.W. Verbiest et al., The international pulsar timing array: first data release, Mon. Not. Roy. Astron. Soc. 458 (2016) 1267 [arXiv: 1602.03640] [INSPIRE].

[31] G. Janssen et al., Gravitational wave astronomy with the SKA, PoS(AASKA14) 037 [arXiv: 1501.00127] [INSPIRE].

[32] R. Zhou, W. Cheng, X. Deng, L. Bian and Y. Wu, Electroweak phase transition and Higgs phenomenology in the Georgi-Machacek model, JHEP 01 (2019) 216 [arXiv:1812.06217] [INSPIRE].

[33] L. Bian, Y. Wu and K.-P. Xie, Electroweak phase transition with composite Higgs models: calculability, gravitational waves and collider searches, JHEP 12 (2019) 028 [arXiv: 1909.02014] [INSPIRE].

[34] L. Bian and X. Liu, Two-step strongly first-order electroweak phase transition modified FIMP dark matter, gravitational wave signals and the neutrino mass, Phys. Rev. D 99 (2019) 055003 [arXiv: 1811.03279] [INSPIRE].

[35] L. Bian and Y.-L. Tang, Thermally modified sterile neutrino portal dark matter and gravitational waves from phase transition: The Freeze-in case, JHEP 12 (2018) 006 [arXiv: 1810.03172] [INSPIRE].

[36] W. Chao, H.-K. Guo and J. Shu, Gravitational wave signals of electroweak phase transition triggered by dark matter, JCAP 09 (2017) 009 [arXiv:1702.02698] [INSPIRE]. 
[37] S. Profumo, M.J. Ramsey-Musolf, C.L. Wainwright and P. Winslow, Singlet-catalyzed electroweak phase transitions and precision Higgs boson studies, Phys. Rev. D 91 (2015) 035018 [arXiv: 1407.5342] [INSPIRE].

[38] S.R. Coleman and E.J. Weinberg, Radiative corrections as the origin of spontaneous symmetry breaking, Phys. Rev. D 7 (1973) 1888 [INSPIRE].

[39] M. Quirós, Finite temperature field theory and phase transitions, hep-ph/9901312 [INSPIRE].

[40] C.L. Wainwright, CosmoTransitions: computing cosmological phase transition temperatures and bubble profiles with multiple fields, Comput. Phys. Commun. 183 (2012) 2006 [arXiv: 1109.4189] [INSPIRE].

[41] A. Ilnicka, T. Robens and T. Stefaniak, Constraining extended scalar sectors at the LHC and beyond, Mod. Phys. Lett. A 33 (2018) 1830007 [arXiv:1803.03594] [inSPIRE].

[42] H.H. Patel and M.J. Ramsey-Musolf, Baryon washout, electroweak phase transition and perturbation theory, JHEP 07 (2011) 029 [arXiv:1101.4665] [INSPIRE].

[43] R. Zhou and L. Bian, Baryon asymmetry and detectable gravitational waves from electroweak phase transition, arXiv:2001.01237 [INSPIRE].

[44] R. Zhou, L. Bian and H.-K. Guo, Probing the electroweak sphaleron with gravitational waves, arXiv:1910.00234 [INSPIRE].

[45] X. Gan, A.J. Long and L.-T. Wang, Electroweak sphaleron with dimension-six operators, Phys. Rev. D 96 (2017) 115018 [arXiv:1708.03061] [InSPIRE].

[46] M. Dine, P. Huet and R.L. Singleton, Jr., Baryogenesis at the electroweak scale, Nucl. Phys. B 375 (1992) 625 [INSPIRE].

[47] I. Affleck, Quantum statistical metastability, Phys. Rev. Lett. 46 (1981) 388 [InSPIRE].

[48] A.D. Linde, Decay of the false vacuum at finite temperature, Nucl. Phys. B 216 (1983) 421 [Erratum ibid. B 223 (1983) 544] [INSPIRE].

[49] A.D. Linde, Fate of the false vacuum at finite temperature: theory and applications, Phys. Lett. B 100 (1981) 37.

[50] C. Caprini et al., Science with the space-based interferometer eLISA. Part II. Gravitational waves from cosmological phase transitions, JCAP 04 (2016) 001 [arXiv:1512.06239] [INSPIRE].

[51] P.J. Steinhardt, Relativistic detonation waves and bubble growth in false vacuum decay, Phys. Rev. D 25 (1982) 2074 [INSPIRE].

[52] M. Hindmarsh, S.J. Huber, K. Rummukainen and D.J. Weir, Gravitational waves from the sound of a first order phase transition, Phys. Rev. Lett. 112 (2014) 041301 [arXiv: 1304.2433] [INSPIRE].

[53] M. Hindmarsh, S.J. Huber, K. Rummukainen and D.J. Weir, Numerical simulations of acoustically generated gravitational waves at a first order phase transition, Phys. Rev. D 92 (2015) 123009 [arXiv:1504.03291] [INSPIRE].

[54] J.R. Espinosa, T. Konstandin, J.M. No and G. Servant, Energy budget of cosmological first-order phase transitions, JCAP 06 (2010) 028 [arXiv: 1004.4187] [INSPIRE].

[55] C. Caprini, R. Durrer and G. Servant, The stochastic gravitational wave background from turbulence and magnetic fields generated by a first-order phase transition, JCAP 12 (2009) 024 [arXiv: 0909.0622] [INSPIRE].

[56] A. Vilenkin and E.P.S. Shellard, Cosmic strings and other topological defects, Cambridge University PRess, Cambridge U.K. (2000). 
[57] H. Hattori, T. Kobayashi, N. Omoto and O. Seto, Entropy production by domain wall decay in the NMSSM, Phys. Rev. D 92 (2015) 103518 [arXiv:1510.03595] [INSPIRE].

[58] T. Hiramatsu, M. Kawasaki and K. Saikawa, On the estimation of gravitational wave spectrum from cosmic domain walls, JCAP 02 (2014) 031 [arXiv:1309. 5001] [INSPIRE].

[59] K. Kadota, M. Kawasaki and K. Saikawa, Gravitational waves from domain walls in the next-to-minimal supersymmetric standard model, JCAP 10 (2015) 041 [arXiv:1503.06998] [INSPIRE].

[60] M. Kawasaki, K. Kohri and T. Moroi, Hadronic decay of late-decaying particles and Big-Bang nucleosynthesis, Phys. Lett. B 625 (2005) 7 [astro-ph/0402490] [INSPIRE].

[61] M. Kawasaki, K. Kohri and T. Moroi, Big-Bang nucleosynthesis and hadronic decay of long-lived massive particles, Phys. Rev. D 71 (2005) 083502 [astro-ph/0408426] [INSPIRE].

[62] K. Saikawa, A review of gravitational waves from cosmic domain walls, Universe 3 (2017) 40 [arXiv: 1703.02576] [INSPIRE].

[63] A. Klein et al., Science with the space-based interferometer eLISA: Supermassive black hole binaries, Phys. Rev. D 93 (2016) 024003 [arXiv:1511.05581] [INSPIRE].

[64] V. Corbin and N.J. Cornish, Detecting the cosmic gravitational wave background with the big bang observer, Class. Quant. Grav. 23 (2006) 2435 [gr-qc/0512039] [INSPIRE].

[65] H. Kudoh, A. Taruya, T. Hiramatsu and Y. Himemoto, Detecting a gravitational-wave background with next-generation space interferometers, Phys. Rev. D 73 (2006) 064006 [gr-qc/0511145] [INSPIRE].

[66] DECIGO working group], Space gravitational wave detector DECIGO/pre-DECIGO, Proc. SPIE 10562 (2017) 105623T.

[67] TianQin collaboration, TianQin: a space-borne gravitational wave detector, Class. Quant. Grav. 33 (2016) 035010 [arXiv:1512.02076] [INSPIRE].

[68] X. Gong et al., Descope of the ALIA mission, J. Phys. Conf. Ser. 610 (2015) 012011 [arXiv: 1410.7296] [INSPIRE].

[69] N.S. Manton, Topology in the Weinberg-Salam theory, Phys. Rev. D 28 (1983) 2019 [INSPIRE].

[70] F.R. Klinkhamer and N.S. Manton, A saddle point solution in the Weinberg-Salam theory, Phys. Rev. D 30 (1984) 2212 [INSPIRE].

[71] M.E.R. James, The sphaleron at nonzero Weinberg angle, Z. Phys. C 55 (1992) 515 [INSPIRE].

[72] F.R. Klinkhamer and R. Laterveer, The sphaleron at finite mixing angle, Z. Phys. C 53 (1992) 247 [INSPIRE].

[73] A. Alves, T. Ghosh, H.-K. Guo and K. Sinha, Resonant di-Higgs production at gravitational wave benchmarks: a collider study using machine learning, JHEP 12 (2018) 070 [arXiv: 1808.08974] [INSPIRE]. 